\title{
Cancer-related fatigue, the role of adrenal suppression and steroids: reply to the comments of Eren et al.
}

\author{
Sriram Yennurajalingam • Eduardo Bruera
}

Received: 19 May 2014 / Accepted: 21 July 2014 / Published online: 31 July 2014

(C) Springer-Verlag Berlin Heidelberg 2014

We like to thank Eren et al. [1] for their thoughtful comments regarding our recent paper [2] titled "Reduction of cancerrelated fatigue with dexamethasone: a double-blind, randomized, placebo-controlled trial in patients with advanced cancer."

The mechanism of cancer-related fatigue in advanced cancer is usually complex and multifactorial [3]. The authors claim that in some cases, cancer-related fatigue may be due to iatrogenic effect in which adrenergic insufficiency induced by antiemetic doses of corticosteroids may have causative role. This is an interesting hypothesis that needs to be tested. However, it is important to know that cancer-related fatigue occurs in high frequency and severity in cancer patients who have not received corticosteroids. The use of an extremely low dose of corticosteroids has not been tested and may not be beneficial in reducing fatigue in such patients [3-5]. More research is necessary to determine the longterm safety and efficacy of dexamethasone for cancerrelated fatigue.

Acknowledgments Sriram Yennurajalingam is supported in part by an American Cancer Society Grant entitled Research Scholar Grant for
Independent Investigators Multimodal Study for Cancer Related Fatigue (Grant No. RSG-11-170-01-BCM).

Conflict of interest The authors indicate no potential conflicts of interest.

\section{References}

1. Eren OO, Ozturk MA, Oyan B 2014 Cancer-related fatigue: can it be due to adrenal suppression secondary to high-dose steroids used as antiemetic? Support Care Cancer. (in press).

2. Yennurajalingam S, Frisbee-Hume S, Palmer JL et al (2013) Reduction of cancer-related fatigue with dexamethasone: a doubleblind, randomized, placebo-controlled trial in patients with advanced cancer. J Clin Oncol 31:3076-3082

3. Yennurajalingam S, Bruera E (2007) Palliative management of fatigue at the close of life. JAMA 297:295-304

4. Metz CA, Robustelli Della Cuna G, Pellegrini A et al (1989) Effect of methylprednisolone sodium succinate on quality of life in preterminal cancer patients: a placebo-controlled, multicenter study. Eur J Cancer Clin Oncol 25:1817-1821

5. Bruera E, Roca E, Cedaro L et al (1985) Action of oral methylprednisolone in terminal cancer patients. Cancer Treat Rep 69:751-754
S. Yennurajalingam $\cdot$ E. Bruera

The University of Texas MD Anderson Cancer Center, Houston, USA

S. Yennurajalingam $(\bowtie) \cdot$ E. Bruera

Department of Palliative Care \& Rehabilitation Medicine, UT MD

Anderson Cancer Center, Unit 1414, 1515 Holcombe Blvd.,

Houston, TX 77030, USA

e-mail: syennu@mdanderson.org 